\title{
Combining Clustering Algorithms For Provide Marketing Policy in Electronic STORES
}

\author{
Farhad Soleimanian Gharehchopogh ${ }^{1}$, Yasin Rahimpour ${ }^{2}$ and \\ Seyyed Reza Khaze ${ }^{3}$ \\ ${ }^{1}$ Department of Computer Engineering, Urmia Branch, Islamic Azad University, \\ Urmia, Iran \\ ${ }^{2}$ Department of Computer Engineering, Science and Research Branch, \\ Islamic Azad University, West Azerbaijan, Iran \\ ${ }^{3}$ Department of Computer Engineering, Dehdasht Branch, \\ Islamic Azad University, Iran,
}

\begin{abstract}
One of the main challenges of electronic market is how to attract customers as various products and customers' preferences effect widely on it. In this paper, we have started to categorize electronic markets customers using k-means, En, Farthest first algorithms to determine marketing policies and customer satisfaction and indicated that each algorithm either cover the weakness of cluster analyzing of other algorithms for some customers or integrated data of all algorithm analyses which provided detailed results of customers' behavioral methods and its relation with their shopping basket could finally lead us to more income and increased productivity using obtained analyses.
\end{abstract}

\section{KEYWORDS}

Clustering, E-marketing, EM algorithm, k-mean algorithm, Farthestfirst algorithm

\section{INTRODUCTION}

Due to the increasing growth of E-commerce in internet world which resulted in commercial affairs facility, costs decrease and sales medium eliminating, the competition in this market wouldn't be so easy and require performing a series of activities which lead to the promotion in this competitive market. The main element of E-commerce in internet is how to attract customer. Certainly, customer clustering will provide an opportunity to more profit and success in marketing in such websites. In clustering the customers of E-commerce websites, the customers buying data and features are collected and stored in databases which can be used them to get the best strategy in the market. So, the main goal of this paper is to provide customer clustering and analyses to get the best strategy on available data in each clustering.

One of the main challenges of electronic market is how to attract customers as various products and customers' preferences effect widely on it. To do so, we need methods such as clustering to categorize the data rapidly. Along with, confidence is also another important factor which highly effected marketing [1]. 


\section{Clustering Application in E-Store Marketing}

Clustering is one of the sub-categories of data mining $[2,3]$ and is a process in which the similar samples are divided into groups called cluster [4]. Each cluster includes samples in which the members are similar to each other and different with the available samples of other groups [5]. Due to the clustering capabilities in marketing, it is developed to provide more profit to customers. E-commerce websites is a field in which marketing plays an important role in increasing incomes.

To attract more customers, it must be used modern methods such as clustering to increase income rate [6]. In fact, clustering make the websites capable to identify their customers and support them to be successful in obtaining profit and dealing with their competitors. As these websites are designed to develop their strategies and policies considering determined clusters, it would be a defined and specified rating to develop target customers [7].

\section{Customer electronics stores Clustering: A CaSe Study}

In this paper, to represent the clustering applications in relationship among customers behavioral methods while buying and their buying basket and also in determining supportive policies to increase profit and productivity based on similarities and differences of various groups of customers, we begin to cluster customers of Chare E-shopping in Urmia city [8]. Firstly, there were a lot of parameters about customers behavioral and favorite methods in which some of them such as gender, education, occupation, income, type of buying and satisfaction scale are selected. By gathering the noted parameters data from 100 customers of Chare e-shopping which is available in Table 1, we begin to cluster customers and provide analyses and results in following paragraphs.

Table1. Data related to 100 customers of E-shops in Urmia City

\begin{tabular}{|c|c|c|c|c|c|c|}
\hline sex & age & Level of Education & Job & income & Activity & Satisfaction \\
\hline MALE & 43 & Master's Degree licence & Employee & high & $\begin{array}{c}\text { software \& } \\
\text { hardware }\end{array}$ & Yes \\
\hline MALE & 56 & under diploma & Free & high & Audio and video & Yes \\
\hline MALE & 20 & diploma & Unemployed & low & $\begin{array}{c}\text { software \& } \\
\text { hardware }\end{array}$ & No \\
\hline MALE & 35 & Bachelor & Unemployed & low & Multimedia & Yes \\
\hline MALE & 23 & associate degree & Unemployed & low & $\begin{array}{c}\text { software \& } \\
\text { hardware }\end{array}$ & Yes \\
\hline MALE & 21 & diploma & Unemployed & low & Multimedia & No \\
\hline MALE & 28 & Master's Degree licence & Free & medium & Book & Yes \\
\hline MALE & 23 & Bachelor & Free & low & Multimedia & Yes \\
\hline
\end{tabular}


International Journal of Programming Languages and Applications ( IJPLA ) Vol.4, No.1, January 2014

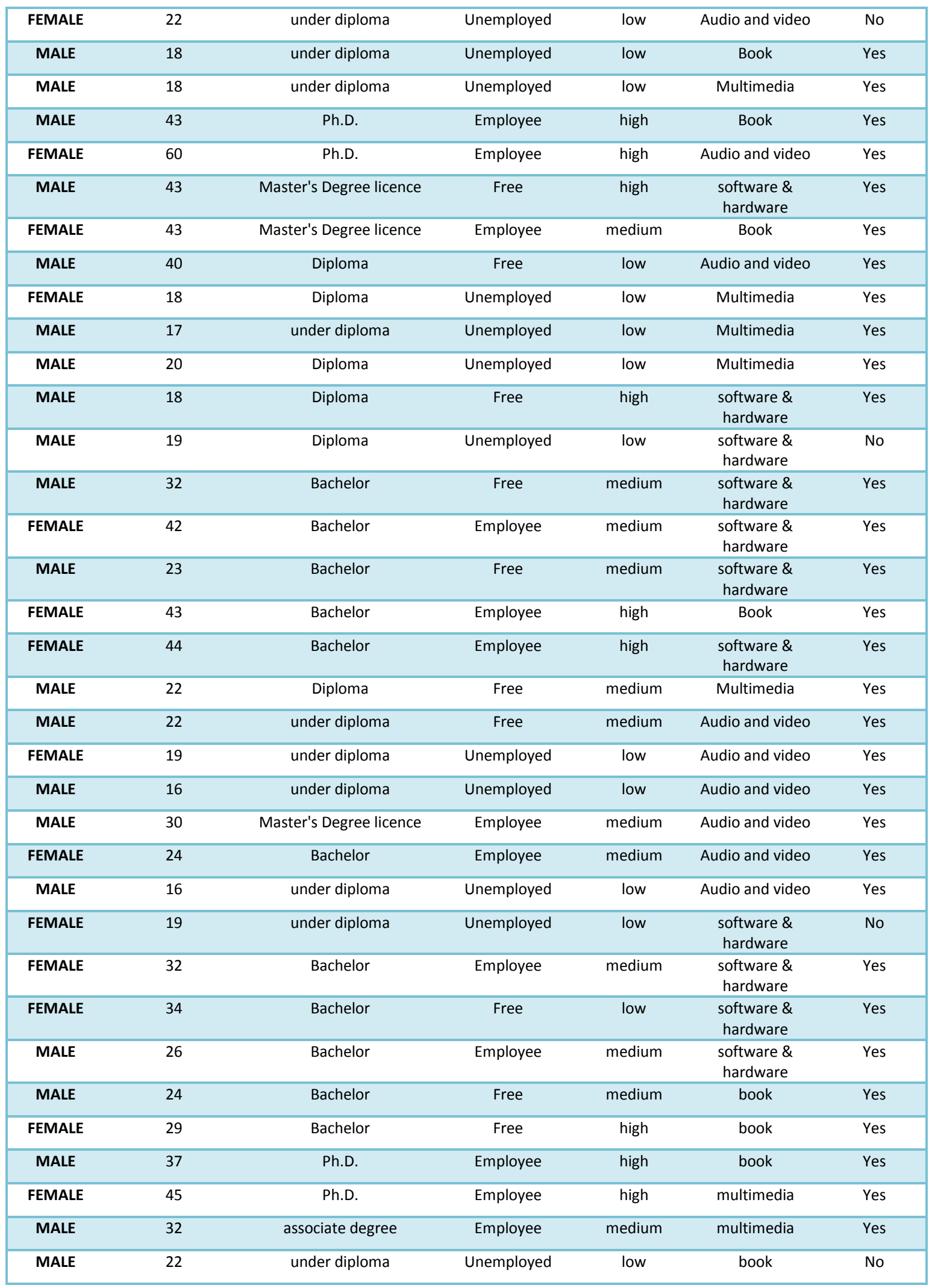


International Journal of Programming Languages and Applications ( IJPLA ) Vol.4, No.1, January 2014

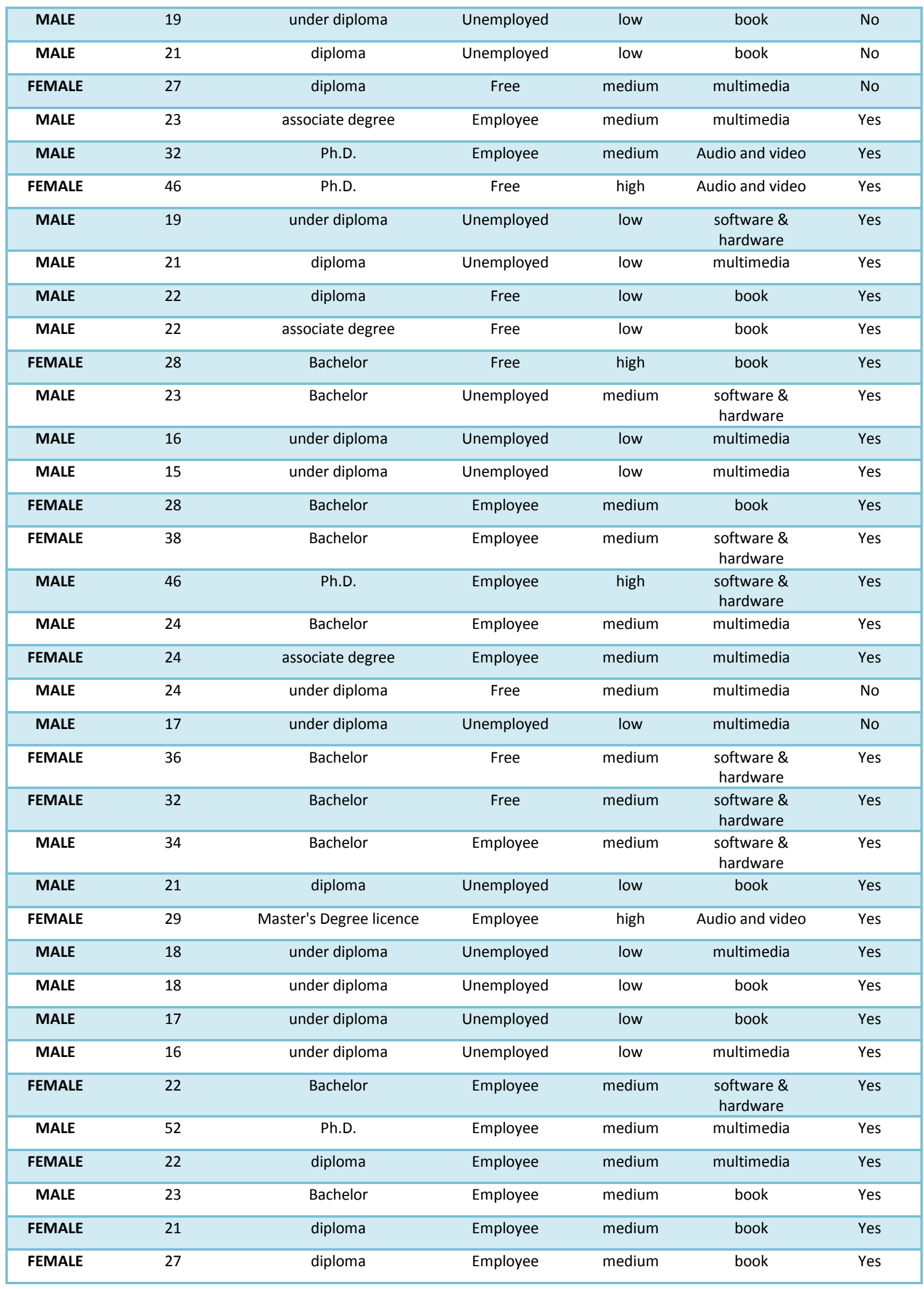


International Journal of Programming Languages and Applications ( IJPLA ) Vol.4, No.1, January 2014

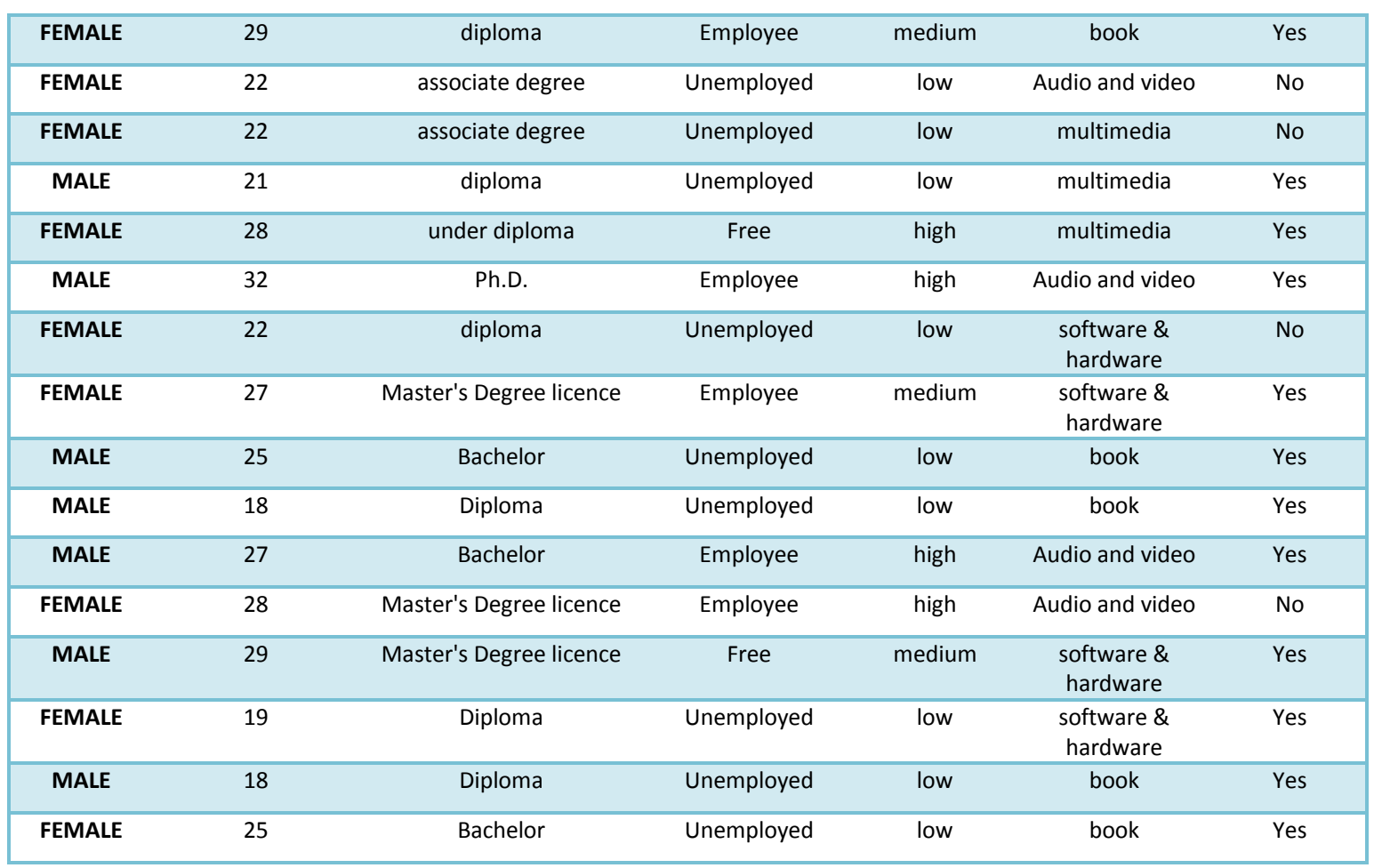

\section{CUSTOMERS CLUSTERING USING DATA MINING ALGORITHMS}

EM is one of the assigned algorithms of clustering and can determine how clusters can create cross-validation. EM algorithm is used to find maximum possible parameters of statistical model in equations which aren't directly solvable [9]. These models usually include potential variables to increase unknown parameters. It means that either there is lost scales among data or the model can be easily adjusted if it is supposed that there are additional data in unseen points. We, in this paper, assign a possible distribution using this algorithm and indicate the possibility of belonging to each customer cluster. K-means is one of the most popular and simple algorithms which simply solve clustering problem [10]. Here, central $\mathrm{K}$ is defined for each cluster of customers. These centers must be selected with high accuracy [11]. Due to the features of this algorithm, the numbers of customer clusters are determined in advance, and then each customer (in the case of similarity) is placed in determined cluster after comparing to customer cluster center. Here, the customers are divided in 4 clusters because if the different centers are selected, it might be created different results. So, the best way to choose centers is that they have the maximum distance from each other. In the next stage, the most similar customer to the cluster centers is assigned to that one. In the next stage, the customers' clusters centers are updated and then the previous stage is repeated as far as no changes occur in cluster. In this case, the customers clustering are finished. Farthestfirst algorithm is another type of k-means which determine the location of cluster center in the farthest point than the available clusters centers [12].

In following, using clustering algorithms to cluster customers, firstly one of the customers is considered as the first cluster center and the next customer as the farthest to the first center and then the third one for the farthest customer to two other customer centers. The final clustering includes the noted stages repetition. 


\section{DISCUSSION}

We, in this paper, use weka tools which are open and free source tool to implement data mining projects [13],[14]. The used k-means results are indicated in Table 2 using this tool to customer clustering.

Table2. Data related to clustering using k-means algorithm

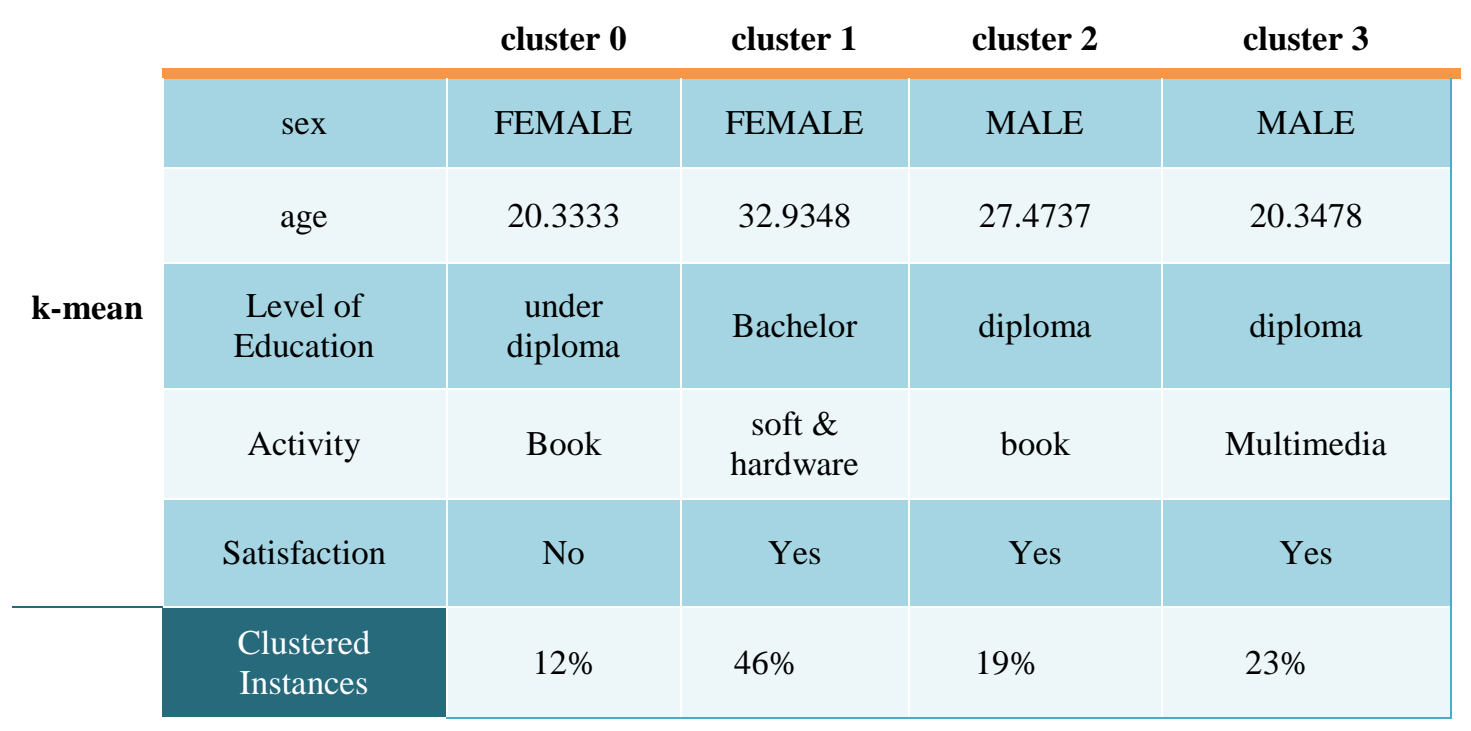

As it is shown, in obtained clusters of -means algorithm, it is assigned clusters 0 and 1 to females and 2 and 3 to males, respectively. Cluster 0 indicated that the women with average 20 years old were mainly under diploma degree, bought books and were displeased about it. The obtained results of Cluster 1 also indicated that women with average 32 years old were bachelor and bought software and hardware and had relative satisfaction about it.

As this cluster includes $46 \%$ of sample community, it can be considered marketing policies and customer attracting regarding the requirements of this group of customers and tried to keep their satisfaction. It can also be modified dissatisfaction rate of buying books via customers' feedback. By gathering their comments, it can be provide their desirable books based on age range of buyers and promote the satisfaction rate. The obtained results of Cluster 2 and 3 indicated that the provided books in shop is according to men needs and had medium scientific level. It was also proper for average 27 years old. The provided multimedia is identified appropriately and this approach can be followed in the shop due to its cheerful and youth favorite products.

We also begin to customer clustering using EM algorithm. The results are indicated in Table 3. 
International Journal of Programming Languages and Applications ( IJPLA ) Vol.4, No.1, January 2014

Table3. Data related to clustering using EM algorithm

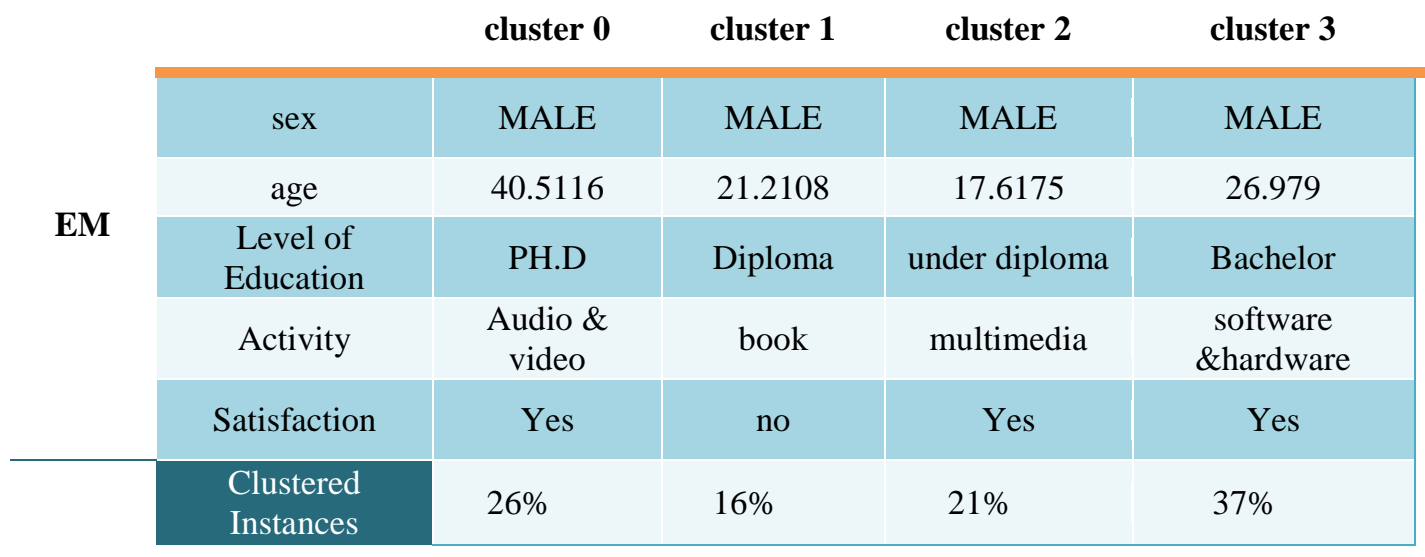

The results of this algorithm clustering allocate to the men which brings advantages and challenges as well. The advantage will be that it provides more detailed results about this gender. The challenge is the lack of marketing and the need to do it for females. So, it can be optimally used this clustering to determine marketing policies of male customers. The results indicated that men with average 40 years old were mostly tend to buy audio and video products and as they average grade were $\mathrm{PhD}$, so they had high satisfaction. The obtained data indicated that they had relative satisfaction about provided products in the market and this approved that these products had high quality.

The youth men who tend to buy books with average 20 years old have shown dissatisfaction. The similar one is also obtained in results of K-means algorithm which provided for women with the same age range. Clearly, due to the obtained results, it must be reviewed about the provided books appropriate to the different age range in the market. In clusters 2 and 3, it is also shown that either men or women feel satisfied about the provided software and hardware products. Considering the results of EM and K-means algorithms, the buying average rates of these products belong to those who had bachelor degree. FM algorithm is also demonstrated the obtained results of 17-20 age range for men. These results have shown that the multimedia products of this shop provided full satisfaction for the customers who had mean degree. The obtained results of Farthestfrirst have been solved some challenges of other algorithms in customers' clustering. The obtained results are indicated in Table 4.

Table 4: data related to clustering using Farthestfirst algorithm

\begin{tabular}{|c|c|c|c|c|c|}
\hline \multirow{6}{*}{ farthestfirst } & & cluster 0 & cluster 1 & cluster 2 & cluster 3 \\
\hline & sex & FEMALE & MALE & FEMALE & MALE \\
\hline & age & 22 & 52 & 29 & 40 \\
\hline & $\begin{array}{c}\text { Level of } \\
\text { Education }\end{array}$ & $\begin{array}{l}\text { associate } \\
\text { degree }\end{array}$ & Ph.D. & bachelor & diploma \\
\hline & Activity & $\begin{array}{l}\text { Audio \& } \\
\text { video }\end{array}$ & multimedia & book & Audio \& video \\
\hline & Satisfaction & No & yes & Yes & Yes \\
\hline & $\begin{array}{l}\text { Clustered } \\
\text { Instances }\end{array}$ & $17 \%$ & $27 \%$ & $26 \%$ & $30 \%$ \\
\hline
\end{tabular}


The clustering we have had with previous algorithms has brought ambiguity for young women customers who proposed to by audio and video products in finding marketing policies type. By using achieved results of farthestfirst algorithm, it is shown that women with average 22 years old feel dissatisfied about provided audio and video products in the market. It is also indicated that women who averaged 29 years old purchase book and feel satisfied about it. It must be note that the average grade of this cluster was bachelor. The men with average 52 years old begin to purchase multimedia products and feel satisfied about it. Men with diploma who bought audio and video products had average 40 years old and feel satisfied. The achieved data of 3 algorithms results are shown in Table 5.

Table5. Analyzed data of customer clustering

\begin{tabular}{|c|c|c|}
\hline Type of product & Analysis & Analytical results \\
\hline $\begin{array}{l}\text { Audio and Video } \\
\text { (Normal quality for playback on } \\
\text { Mobile) }\end{array}$ & Gender-based & $\begin{array}{l}\text { Among people at all levels of school satisfaction } \\
\text { there, but there is dissatisfaction among young } \\
\text { women. Therefore take gender-specific and } \\
\text { product-related men. }\end{array}$ \\
\hline Hardware and Software & education-based & $\begin{array}{l}\text { Most applicants for such products have a bachelor's } \\
\text { degree and are satisfied with the purchase. This } \\
\text { product has not bought the other Degrees }\end{array}$ \\
\hline Book & Age-based & $\begin{array}{l}\text { Who range in age from } 20 \text { to } 21 \text { and have put them } \\
\text { under graduate degree has been reluctant to } \\
\text { purchase books. There is no difference between } \\
\text { men and women. People in the age range } 27 \text { to } 29 \\
\text { years have been, men with diploma And women } \\
\text { with bachelor's degrees, have been satisfied from } \\
\text { buying books. }\end{array}$ \\
\hline $\begin{array}{l}\text { Multimedia } \\
\text { ( high quality for playback on } \\
\text { computer) }\end{array}$ & Gender-based & $\begin{array}{l}\text { None of the clusters, buy Multimedia has been } \\
\text { done by women. All purchases from the monopoly } \\
\text { of men And in all age ranges and for all } \\
\text { qualifications have satisfied. }\end{array}$ \\
\hline
\end{tabular}

\section{CONCLuSiONS}

Each clustering algorithms are solely capable of focusing on particular parts of customers' data in electronic shops. This focus brings better and more detailed results to the same parts. Meanwhile, in analysing other parts, due to the lack of clustering analyses, it brings challenges to them. So, each algorithm is capable of doing detailed analyses of some parts of customers' data. To provide comprehensive results and clustering analyses, it must be used several integrated and clustering algorithms. We, in this paper, investigate different types of methods and clustering algorithms. Finally, by using K-means, farthest first, EM samples of customers of an E-commerce websites, we made clustering vie Weka software. 
We indicated that each algorithm covers the clustering analyses weaknesses of other algorithms for some customers. The integrated data of all algorithms analyses brings detailed results from customers' behavioural method and its relation with shopping basket as well. So, by using integrated collective data, it can be determined marketing policies and customer satisfaction appropriate to all customers' clustering and their orientation which finally lead to increased productivity and incomes.

\section{REFERENCES}

[1] Sohrabi S., Nikkhahan B., Khoshalhan, F.(2008), Customer clustering based on trust formation factor with Genetic Algorithm, International Conference of Innovation in Redefining Business Horizons , IMT Ghaziabad, India

[2] Khalifehlou, Z. A., \& Gharehchopogh, F. S. (2012). A Survey of Data Mining Techniques in Software Cost Estimation. AWERProcedia Information Technology and Computer Science, 1.

[3] Khalifelu, Z. A., \& Gharehchopogh, F. S. (2012). Comparison and evaluation of data mining techniques with algorithmic models in software cost estimation. Procedia Technology, 1, 65-71.

[4] Witten, I. H., Frank, E., \& Hall, M. A. (2011). Data Mining: Practical Machine Learning Tools and Techniques: Practical Machine Learning Tools and Techniques. Elsevier.

[5] Gorunescu, F. (2011). Data Mining: Concepts, models and techniques (Vol. 12). Springer.

[6] Wu, R. S., \& Chou, P. H. (2011). Customer segmentation of multiple category data in e-commerce using a soft-clustering approach. Electronic Commerce Research and Applications, 10(3), 331-341.

[7] Stefanowski, J., \& Weiss, D. (2003). Carrot2 and language properties in web search results clustering. In Advances in Web Intelligence (pp. 240-249). Springer Berlin Heidelberg.

[8] http://www.chare.ir/ (L.A:06/20/2013)

[9] Dempster, A.P.; Laird, N.M.; Rubin, D.B. (1977). "Maximum Likelihood from Incomplete Data via the EM Algorithm". Journal of the Royal Statistical Society. Series B (Methodological) 39 (1): 1-38.

[10] Gharehchopogh, F. S., Jabbari, N., \& Azar, Z. G. (2012). Evaluation of Fuzzy K-Means and K-Means Clustering Algorithms in Intrusion Detection Systems. International Journal of Scientific \& Technology Research, 1(11).

[11] Pelleg, D., \& Moore, A. W. (2000, June). X-means: Extending K-means with Efficient Estimation of the Number of Clusters. In ICML (pp. 727-734).

[12] Hochbaum, D. S., \& Shmoys, D. B. (1985). A best possible heuristic for the k-center problem. Mathematics of operations research, 10(2), 180-184.

[13] Gharehchopogh, F.S., \& Khaze, S.R. (2012). Data Mining Application for Cyber Space Users Tendency in Blog Writing: A Case Study. International Journal of Computer Applications, 47(18), 40-46.

[14] Berry, M. J., \& Linoff, G. (1997). Data mining techniques: For marketing, sales, and customer support. John Wiley \& Sons, Inc.

\section{Authors}

Farhad Soleimanian Gharehchopogh is currently $\mathrm{Ph} . \mathrm{D}$. candidate in department of computer engineering at Hacettepe University, Ankara, Turkey. And he works an honour lecture in computer engineering department, science and research and Urmia branches, Islamic Azad University, West Azerbaijan, Iran. He is a member of editorial board and review board in many international journals and international Conferences. His interested research areas are in the Operating Systems, Software Cost Estimation, Data Mining and Machine Learning techniques and Natural Language Processing. For more information

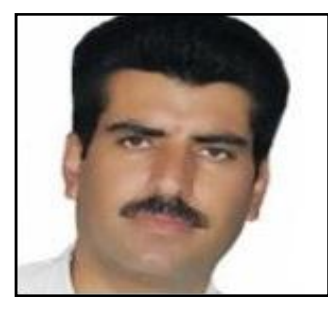
please visit www.soleimanian.net 
International Journal of Programming Languages and Applications ( IJPLA ) Vol.4, No.1, January 2014

Yasin Rahimpour is a M.Sc. student in Computer Engineering Department, Science and Research Branch, Islamic Azad University, West Azerbaijan, Iran. His interested research areas are Meta Heuristic Algorithms, Data Mining and Machine learning Techniques.

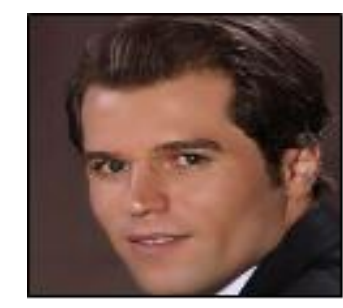

Seyyed Reza Khaze is a Lecturer and Member of the Research Committee of the Department of Computer Engineering, Dehdasht Branch, Islamic Azad University, Iran. He is a Member of Editorial Board and Review Board in Several International Journals and National Conferences. His interested research areas are in the Software Cost Estimation, Machine learning, Data Mining, Optimization and Artificial Intelligence.

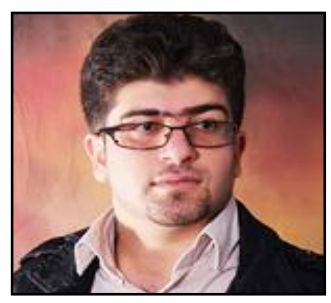

